\title{
Somatotopic Activation in the Human Trigeminal Pain Pathway
}

\author{
Alex F. M. DaSilva, ${ }^{1}$ Lino Becerra, ${ }^{1}$ Nikos Makris, ${ }^{2}$ Andrew M. Strassman, ${ }^{3}$ R. Gilberto Gonzalez, ${ }^{1}$ \\ Nina Geatrakis, ${ }^{1}$ and David Borsook ${ }^{1}$ \\ ${ }^{1}$ Center for Pain Functional Neuroimaging and Therapy Research, Athinoula Martinos Center for Biomedical Imaging, and \\ 2Department of Radiology, Center for Morphometric Analysis, Department of Neurology, Massachusetts General Hospital, \\ Harvard Medical School, Boston, Massachusetts 02129, and '3Department of Anesthesia and Critical Care, Beth Israel- \\ Deaconess Medical Center, Harvard Medical School, Boston, Massachusetts 02215
}

Functional magnetic resonance imaging was used to image pain-associated activity in three levels of the neuraxis: the medullary dorsal horn, thalamus, and primary somatosensory cortex. In nine subjects, noxious thermal stimuli $\left(46^{\circ} \mathrm{C}\right)$ were applied to the facial skin at sites within the three divisions of the trigeminal nerve (V1, V2, and V3) and also to the ipsilateral thumb. Anatomical and functional data were acquired to capture activation across the spinothalamocortical pathway in each individual. Significant activation was observed in the ipsilateral spinal trigeminal nucleus within the medulla and lower pons in response to at least one of the three facial stimuli in all applicable data sets. Activation from the three facial stimulation sites exhibited a somatotopic organization along the longitudinal (rostrocaudal) axis of the brain stem that was consistent with the classically described "onion skin" pattern of sensory deficits observed in patients after trigeminal tractotomy. In the

The ability to discriminate and interpret noxious information from the environment is an important component of mammalian survival. Nociceptive information is relayed in a pathway that includes the spinal or medullary dorsal horn, the thalamus, and the primary somatosensory cortex (S1). To date, functional neuroimaging studies in humans have not demonstrated simultaneous activation in this pathway after pain stimuli. The trigeminal system offers unique advantages for studying the central processing of nociceptive information in human subjects, including its large central representation, the cranial location of the secondorder neurons, and a presumed high degree of somatotopy.

Clinical observations indicating a complex organization of the trigeminal nucleus (spV) in humans (Gybels and Sweet, 1989) have been supported by studies in nonhuman primates (Bushnell et al., 1984; Dubner et al., 1989; Maixner et al., 1989; Craig et al., 1999). These studies suggest that the somatotopic representation of the face in the $\mathrm{spV}$ is in an "onion skin" or segmental distribution: the perioral areas are represented rostrally in the nucleus, and more posterior areas of the face are represented more caudally within the spV (Kunc, 1970; Kim et al., 1997).

Received March 25, 2002; revised June 17, 2002; accepted July 1, 2002.

This work was supported by a grant from the MayDay Foundation (New York, NY) to D.B. and in partial support of A.D.'s salary from Capes (Rio De Janeiro, Brazil). We thank our colleagues Dr. Alex Ploghaus, Dr. Christina Borras, Dr. Braden Kuo, and Julie Gostic for helpful input.

Correspondence should be addressed to Dr. David Borsook, Center for Pain Functional Neuroimaging and Therapy Research, Department of Radiology, Massachusetts General Hospital, Building 149, 13th Street, Charlestown, MA 02129. E-mail: borsook@nmr.mgh.harvard.edu.

Copyright (C) 2002 Society for Neuroscience $\quad 0270-6474 / 02 / 228183-10 \$ 15.00 / 0$ thalamus, activation was observed in the contralateral side involving the ventroposteromedial and dorsomedial nuclei after stimulation of the face and in the ventroposterolateral and dorsomedial nuclei after stimulation of the thumb. Activation in the primary somatosensory cortex displayed a laminar sequence that resembled the trigeminal nucleus, with V2 more rostral, V1 caudal, and V3 medial, abutting the region of cortical activation observed for the thumb. These results represent the first simultaneous imaging of pain-associated activation at three levels of the neuraxis in individual subjects. This approach will be useful for exploring central correlates of plasticity in models of experimental and clinical pain.

Key words: pain; functional magnetic resonance imaging; trigeminal system; trigeminal nucleus; noxious heat; spinal nucleus of V; somatotopy; ventrobasal complex; somatosensory cortex

Nociceptive neurons within the $\mathrm{spV}$ send projections to the thalamus (Hu et al., 1981; Williams et al., 1994; Craig et al., 1999; Dostrovsky, 2000). These terminate in the ventroposteromedial (VPM) nucleus of the ventrobasal complex of the thalamus and other more medial nuclei (Dostrovsky and Guilbaud, 1990; Rausell and Jones, 1991; Bushnell et al., 1993; Sherman et al., 1997). Unlike the distinct somatotopic representation of the body in the ventroposterolateral (VPL) nucleus, the somatotopy of facial representation is less distinct (Dostrovsky, 2000). To date, no functional imaging studies have demonstrated that noxious stimulation to the body and face produces distinct patterns of thalamic and cortical activation in the same individuals.

The primary somatosensory cortex (S1) receives noxious and non-noxious somatosensory inputs from the thalamus (Chudler et al., 1990; Rausell and Jones, 1991) and encodes information related to the discriminative aspects of pain (Bushnell et al., 1999, Hofbauer et al., 2001). Anatomical data in primates has defined a complex somatotopic representation of the face in S1 (Manger et al., 1996; Jain et al., 2001). In humans, functional magnetic resonance imaging (fMRI) data suggest that the homuncular representation of the face in S1 is inverted (Servos et al., 1999), contradicting the classical view based on anatomical studies (Penfield and Rasmussen, 1950).

Functional imaging studies in humans have identified specific pain-associated regions of activation in the thalamus or the cerebral cortex (Talbot et al., 1991; Coghill et al., 1994, 1999, 2001; Casey et al., 1996; Svensson et al., 1997; Davis et al., 1998; Apkarian et al., 2000a; Becerra et al., 2001). Positron emission 
A. Facial Map

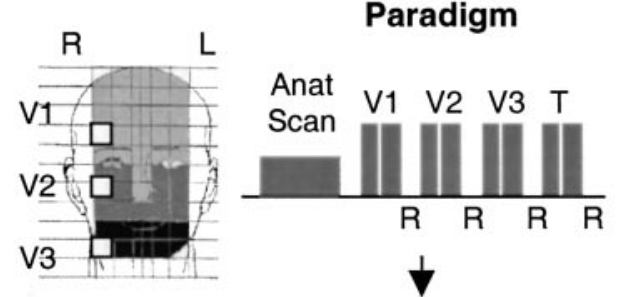

\section{Slice Orientation}

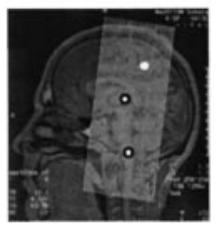

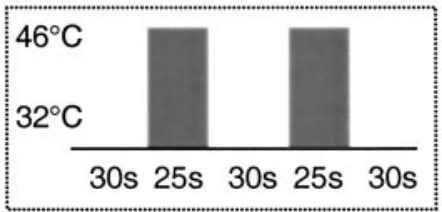

Figure 1. Experimental paradigm. A, Facial map. White squares denote location of the Peltier probe on the face. The three shades of gray differentiate the regions innervated by the V1 (ophthalmic; light gray), V2 (maxillary; medium gray), and V3 (mandibular; dark gray) divisions of the trigeminal nerve. $B$, Stimulus paradigm. After the anatomical scan, the Peltier thermode was applied to the target area within the V1 distribution, and two $25 \mathrm{sec}$ pulses of $46^{\circ} \mathrm{C}$ were given $30 \mathrm{sec}$ apart with a baseline temperature of $32^{\circ} \mathrm{C}$. After a $3 \mathrm{~min}$ interval, the stimuli were repeated with the probe in the $\mathrm{V} 2$ target region. The same procedure was used for the V3 region and finally for the palmar surface of the right thumb $(T)$. After each set of stimuli, subjects rated their pain scores $(R$, visual analog rating of pain level from $0=$ no pain to $10=$ maximum pain imaginable). The time line for stimulus duration and interstimulus interval at each site is indicated within the box. $C$, Slice orientation. Shown is the orientation through the midsagittal section of the whole brain used for these experiments. Slices $(n=30)$ were oriented parallel to the brainstem. The circles show the location of the spV and thalamus and the approximate location of the SI (projected from its lateral location).

tomographic studies examining activity in the trigeminal system after painf ul stimulation (Weiller et al., 1995; Hsieh et al., 1996; May et al., 1998) have reported activation in brainstem structures such as the periaqueductal gray, but not in the trigeminal nucleus. Using structural and functional MRI, we have examined activation after noxious facial stimulation at all three levels of the neuraxis: the spinal trigeminal nucleus, thalamus, and cortex.

\section{MATERIALS AND METHODS}

\section{Human subjects}

The Subcommittee on Human Studies at the Massachusetts General Hospital approved the study, which conformed to the guidelines of the Helsinki agreement and the International Association for the Study of Pain on human experimentation. Nine healthy right-handed male volunteers (age, $29.4 \pm 5.05$ years) were recruited to the study.

Study subjects were pain-free and had no significant medical history, including psychiatric illness or facial or dental pain, and were not taking any medications. Before the experiment, subjects were informed in detail about the nature of experiment and the temporal sequence of stimulation procedures, including methods of rating the thermal stimuli. Once in the scanner, subjects were asked to remain still for the duration of the experiment and were instructed to close their eyes during acquisition of functional scans. Subjects were informed that they could terminate the experiment at any time.

\section{Thermal stimulation and pain ratings}

A $1.6 \times 1.6 \mathrm{~cm}$ thermode was used to apply thermal stimuli to four stimulation sites in each subject in the following order: three premarked sites in the ophthalmic (V1), maxillary (V2), and mandibular (V3) divisions on the right side of the face (Fig. $1 A$ ) and the palmar surface of the right thumb. Only one site was stimulated at a time. Each site received a single stimulus trial that consisted of two painful heat stimuli of $46^{\circ} \mathrm{C}$ lasting $25 \mathrm{sec}$ each, separated by a $30 \mathrm{sec}$ interval at the baseline temperature of $32^{\circ} \mathrm{C}$ (Fig. $1 B$ ). After each stimulus trial, the subjects rated their pain intensity using a Likert visual analog scale (VAS) of 0 (no pain) to 10 (highest pain imaginable). Stimulus trials at the different sites were separated by intertrial intervals of $3 \mathrm{~min}$.

\section{Scanning}

Subjects were scanned on a Siemens Sonata $1.5 \mathrm{~T}$ system. Imaging for all nine subjects began with a three-plane scout scan. The axial and coronal scouts were used for placement and prescription of a three-dimensional sagittal scan [128 slices; $1.328 \mathrm{~mm}$ thick in plane resolution $1 \times 1 \mathrm{~mm}$; repetition time (TR), $11.1 \mathrm{sec}$; echo time (TE), $4.3 \mathrm{msec}]$. This scan was used for transformation of the forebrain into the Talairach space (Talairach and Tournoux, 1988). fMRI scans were acquired using a gradient echo sequence with TR of $3.5 \mathrm{sec}$, TE of $40 \mathrm{msec}$, and in-plane resolution of $3.125 \mathrm{~mm}$. For each functional run, 45 volumes of 30 slices, $3 \mathrm{~mm}$ thick, were acquired. The slices were oriented in an oblique plane, parallel to the medulla and covering the brainstem and most of the parietal lobes (Fig. 1C).

\section{Data analysis}

The image analysis package MEDx 3.3 (Sensor Systems, Bethesda, MD) was used for all data processing.

\section{Motion correction}

Areas around the cortical and $\mathrm{spV}$ activations were inspected for residual motion by visual animation display of each slice after motion correction.

Motion in the brainstem has two origins: gross head motion and cardiac pulsation. Gross head motion was minimized by restraining the head during scanning. Most motion correction algorithms are based on a 12-parameter, rigid body model (MEDx). Such an approach will emphasize realignment of the cerebrum but not necessarily the brainstem. In these experiments, a strict limit for overall displacement $(1 \mathrm{~mm})$ was implemented to ensure that the motion correction process does not worsen the motion artifacts in the brainstem. Cardiac pulsation worsens partial volume effects; hence slices were prescribed along the medulla axis to reduce these effects, which were further minimized by the in-plane smoothing process (see below).

\section{Spatial filtering}

Gaussian filtering was applied with 6/6/6 mm width. Functional data were then intensity normalized and thresholded. Gaussian filtering was applied at two levels for the individual analysis: $6 / 6 / 6$ and $1.5 / 1.5 / 1 \mathrm{~mm}$. The latter filtering was used to reduce the smearing of the $\mathrm{spV}$ activation by the smoothing process in the analysis of "native" (non-Talairach) scans.

\section{Registration to the atlas}

Individual anatomical and functional data were translated into the $\mathrm{Ta}$ lairach coordinate system (Talairach and Tournoux, 1988). A Talairach average group for the functional and anatomical data was created for each trigeminal branch (V1, V2, and V3) and the thumb.

\section{Group average and individual analysis}

Student $t$ test maps were produced on a voxel-by-voxel basis on the individual and averaged data sets. Transition time points between the baseline and the target temperature were removed from the analysis, leaving for calculation only the steady-state time points for the 32 and $46^{\circ} \mathrm{C}$ conditions. The statistical maps were transformed into $-\log P$ maps, color-coded by magnitude, and rendered over the respective group average anatomical images.

Individual analysis was performed in the original native data sets and in the Talairach-transformed ones.

Statistical thresholds were based on a cluster size approach (Forman et al., 1995). Clusters of at least 10 pixels and $p<0.03$ were selected for activation in the cerebrum and group average data. For native data, processed with a smaller filter width, a cluster size of 3 pixels and $p<$ 0.03 were selected.

\section{Anatomical localization}

S1 and thalamus. Activations in the forebrain were localized using the coplanar stereotaxic atlas of the human brain (Talairach and Tournoux, 1988). Individual activations in the regions of interest in the forebrain were tabulated (allowing the calculation of group means).

Brainstem including $s p V$. The Talairach coordinate system is not specific for the brainstem; pixels observed to be significantly activated in the medulla were also identified and localized separately for each individual. 
Together with the Center for Morphometric Analysis at Massachusetts General Hospital (N. Makris), a self-referential approach for the brainstem was adopted (Kennedy et al., 1997). To localize functional activation within the trigeminal nuclei using MRI, we followed a landmarkbased topographical approach (DaSilva et al., 2001). To this end, the human brainstem has been parcellated into 28 distinct parcellation units (PUs; 14 PUs per side) as follows.

The midbrain can be subdivided into six PUs. A line in the midcollicular axial plane in the superior-inferior direction divides the region into two divisions, rostral and caudal components. Each one of these sectors was subdivided in the anteroposterior direction into three subsectors, resulting in six PUs for the entire midbrain (i.e., anterior, middle, and posterior segments within the rostral and caudal components, respectively). The anterior PUs contain the crus cerebri and the substantia nigra. The division between middle and posterior regions would be done by a coronal plane passing through the cerebral aqueduct. Therefore, the middle PUs would correspond to the midbrain tegmentum, and the posterior PUs would correspond to the midbrain tectum.

A similar approach was used in the pons, which was divided into four PUs. In the superior-inferior direction, the region was divided into two sectors by an axial plane passing through the line that connects the roots of the trigeminal nerve $(\mathrm{V})$ as it enters the pons. Each of these was subdivided in the anteroposterior direction into two subsectors, resulting in four PUs, (i.e., rostral-anterior and posterior and caudal-anterior and posterior). The border between anterior and posterior sectors is the coronal plane, containing the stems of the middle cerebellar peduncles. Thus, anterior PUs contain the crossing transverse pontocerebellar fibers and the descending corticospinal fiber system, whereas the posterior PUs contain the remaining structures and the fourth ventricle pertaining to the pons. The posterior PUs would correspond to the pontine tegmentum, and the anterior regions of interest (ROIs) would correspond to the basal pons.

The medulla was subdivided into four PUs. In the superior-inferior direction, the region was divided into two sectors at the axial plane where the pyramids merge in the midline to decussate. This approximates the floor of the inferior olivary nucleus, therefore, in the rostral and caudal medulla. Each one of these sectors has been subdivided in the anteroposterior direction into two subsectors, resulting in four PUs for the entire medulla (i.e., rostral-anterior and posterior and caudal-anterior and posterior). Anterior PUs contain the pyramids, pyramidal decussation, and inferior olivary nucleus, whereas posterior PUs contain the remaining structures and the fourth ventricle pertaining to the medulla. The division between anterior and posterior regions was defined as a coronal plane passing through the anterolateral sulci of the two sides. Therefore, the anterior PUs correspond to the medullar base, and the posterior PUs correspond to the medullar tegmentum.

The parcellation method described above was used to define the topographic anatomy of the $\mathrm{spV}$. The $\mathrm{spV}$, which comprises oral, interpolar and, caudal nuclei, extends from the lower to mid pons to the upper cervical spinal cord (Fig. $2 A$ ). The rostral end of the $\mathrm{spV}$ is located at the lower midpontine level (Carpenter and Sutin, 1983), adjacent to anterolateral corner of the fourth ventricle in a ventrolateral position. Within the upper and mid portions of the medulla, the $\mathrm{spV}$ maintains its ventrolateral position relative to the fourth ventricle and a medial position to the medial cerebellar peduncle and posterior to the inferior olivary nucleus (Carpenter and Sutin, 1983; Haines, 1994). At the level of the obex, the anatomical topography changes. In the lower medulla (below the obex), the $\mathrm{spV}$ is found approximately equidistant between the pyramidal tract and the gracile nucleus in the dorsoventral extent. At the coronal level of the spinal canal, the $\mathrm{spV}$ is within half distance from the external margin of the medulla. In this parcellation system, the $\mathrm{spV}$ would be included in the following PUs: anterior caudal pons (P2a), posterior caudal pons (P2p), posterior rostral medulla (B1p), and posterior caudal medulla (B2p) (Fig. 2C,D). More specifically, although it will be included entirely within B1p and $\mathrm{B} 2 \mathrm{p}$ and the bulbar level, it is located in the border zone of P2a and P2p.

The individual Talairach activations in the ROIs, including $\mathrm{spV}$, thalamus [dorsomedial (DM), VPM, and VPL], and S1, were accepted only if located no more than three pixels from the coordinates of the peak of activation in each ROI in the group average. This method was used to assess whether group average results were biased by a small number of the subjects. Volumes of activations were determined from ROI cluster size.

\section{RESULTS}

Using the paradigm described above, nine subjects received noxious thermal stimuli $\left(46^{\circ} \mathrm{C}\right)$ to the facial skin at sites within the three divisions of the trigeminal nerve (V1, V2, and V3) and also

\section{A. Trigeminal Nuclei}

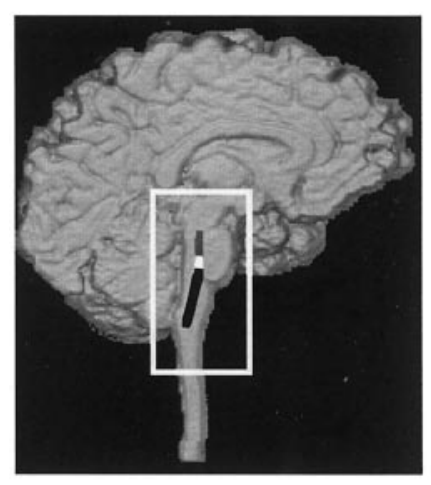

\section{B. Brainstem}

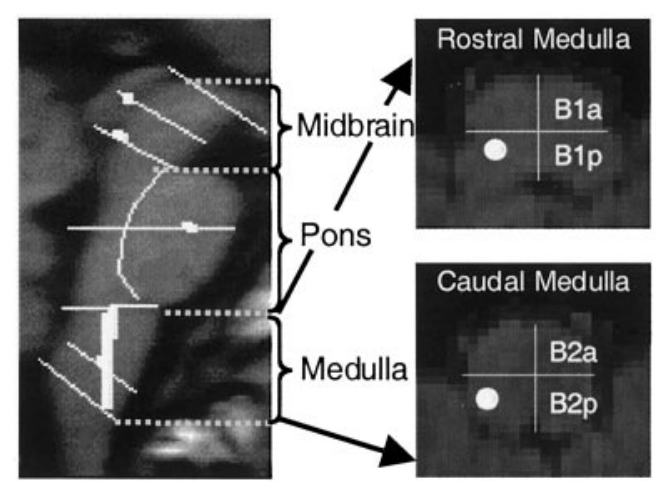

Figure 2. Brainstem parcellation. $A$, Trigeminal nuclei. Shown is the approximate distribution of the trigeminal sensory complex in the dorsal part of the brainstem. Gray, Mesencephalic nucleus; white, main sensory nucleus; black, spinal trigeminal nuclei (oralis, interpolaris, and caudalis). $B$, Parcellation of the brainstem in a sagittal section. To localize functional activation within the trigeminal nuclei using MRI, we followed a landmark-based topographical approach (for details, see Materials and Methods). Each line is defined on MRI-based anatomy that is easily visualized. To this end, the human brainstem has been parcellated into 28 distinct PUs (14 PUs per side) in the midbrain, pons, and medulla. $C$, Parcellation of the medulla. A horizontal section through the rostral and caudal medulla is shown. The medulla has been subdivided into four PUs [rostral-anterior $(B 1 a)$, rostral-posterior $(B 1 p)$, caudal-anterior $(B 2 a)$, caudal-posterior $(B 2 p)]$. See Materials and Methods for details of the parcellation method. Within the medulla, the spinal nucleus of $\mathrm{V}$ is found in the $B 1 p$ and $B 2 p$ posterior components (white circles). D, Activation map. Shown is a statistical map of activation within the B1p segment of the right rostral medulla after a noxious thermal stimulus to the face. Note that this is the region where the $\mathrm{spV}$ is predicted (white circle in $C$ ).

to the ipsilateral thumb. Anatomical and functional data were acquired to capture activation across the spinothalamocortical pathway in each individual. After each stimulus trial, the subjects rated their pain intensity using a Likert VAS of 0 (no pain) to 10 (highest pain imaginable).

\section{Psychophysical results}

VAS scores are shown in Table 1. After thermal stimulation of each division of the face, VAS ratings for the group were $6.16 \pm$ 0.98 for $\mathrm{V} 1,6.64 \pm 0.56$ for $\mathrm{V} 2$, and $6.00 \pm 0.71$ for $\mathrm{V} 3$, respectively, and $5.5 \pm 1.00$ after thermal stimulation to the thumb.

\section{Functional magnetic resonance imaging data}

After thermal stimuli to the face, we examined activation within the $\mathrm{spV}$, thalamus, and S1 cortex for each individual. Group 


\section{Table 1. Individual VAS}

\begin{tabular}{|c|c|c|c|c|c|c|c|c|c|}
\hline \multirow[b]{2}{*}{ Stimulus site } & \multicolumn{7}{|c|}{ Subject } & \multirow[b]{2}{*}{ Mean } & \multirow[b]{2}{*}{$\mathrm{SD}$} \\
\hline & 1 & 2 & 3 & 4 & 5 & 6 & 7 & & \\
\hline Ophthalmic division (V1) & 5 & 7 & 5 & 7 & 7 & 6 & $7 \Delta$ & 6.16 & 0.98 \\
\hline Maxillary division (V2) & 6.5 & 7.5 & 6.5 & 6 & 6 & 7 & 7 & 6.64 & 0.56 \\
\hline Mandibular division (V3) & 6 & $\phi$ & $\phi$ & 7 & 5 & 6 & 6 & 6 & 0.71 \\
\hline Thumb & 4 & $\phi$ & $\phi$ & 6 & $\phi$ & 6 & 6 & 5.5 & 1 \\
\hline
\end{tabular}

$\Delta$, Movement; $\phi$, thermal sensory device malfunction.

\section{Table 2. Group analysis}

\begin{tabular}{|c|c|c|c|c|c|c|}
\hline \multirow[b]{2}{*}{ Stimulus site } & \multirow[b]{2}{*}{ Brain region } & \multicolumn{3}{|c|}{ Talairach coordinates } & \multirow[b]{2}{*}{ Volume $\left(\mathrm{cm}^{3}\right)^{a}$} & \multirow[b]{2}{*}{$p^{b}$} \\
\hline & & $\operatorname{ML}(x)$ & $\mathrm{AP}(y)$ & $\operatorname{SI}(z)$ & & \\
\hline \multirow[t]{5}{*}{ Ophthalmic division (V1) } & $\mathrm{spV}$ & 2 & -36 & -48 & 0.3 & $6.8 \times 10^{-3}$ \\
\hline & Thal & & & & & \\
\hline & VPM/VPL & -18 & -20 & 10 & 0.7 & $2.0 \times 10^{-6}$ \\
\hline & $\mathrm{DM}$ & -2 & -14 & 10 & 0.89 & $6.4 \times 10^{-6}$ \\
\hline & SI & -60 & -22 & 30 & 1.02 & $1.5 \times 10^{-4}$ \\
\hline \multirow[t]{5}{*}{ Maxillary division (V2) } & $\mathrm{spV}$ & 6 & -36 & -38 & 0.42 & $8.8 \times 10^{-4}$ \\
\hline & Thal & & & & & \\
\hline & VPM/VPL & -16 & -18 & 8 & 0.7 & $3.5 \times 10^{-4}$ \\
\hline & $\mathrm{DM}$ & -2 & -16 & 8 & 0.81 & $8.7 \times 10^{-5}$ \\
\hline & SI & -60 & -16 & 16 & 1.7 & $1.4 \times 10^{-7}$ \\
\hline \multirow[t]{5}{*}{ Mandibular division (V3) } & $\mathrm{spV}$ & 4 & -34 & -42 & 0.1 & $3.0 \times 10^{-3}$ \\
\hline & Thal & & & & & \\
\hline & VPM/VPL & -18 & -18 & 8 & 0.61 & $7.1 \times 10^{-5}$ \\
\hline & DM & -4 & -16 & 10 & 0.76 & $8.5 \times 10^{-5}$ \\
\hline & SI & -58 & -18 & 24 & 0.29 & $4.4 \times 10^{-3}$ \\
\hline \multirow[t]{4}{*}{ Thumb } & Thal & & & & & \\
\hline & VPL & -16 & -16 & 10 & 1.01 & $1.0 \times 10^{-5}$ \\
\hline & DM & -8 & -16 & -4 & 0.66 & $1.9 \times 10^{-3}$ \\
\hline & SI & -50 & -26 & 32 & 0.8 & $4.0 \times 10^{-3}$ \\
\hline
\end{tabular}

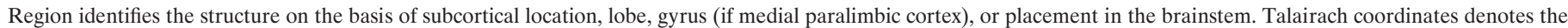

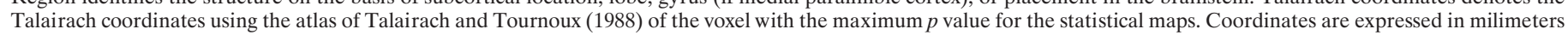
from the anterior commissure. ML, Medial (+)-lateral (-); AP, anterior (+)-posterior (-); SI, superior (+)-inferior $(-)$; Thal, thalamus.

${ }^{a}$ Volume of activation.

${ }^{b}$ Maximum $p$ value for each activated cluster of voxels on the statistical map.

(Table 2) and individual (Table 3) results for activation within these structures are provided below. Of the nine subjects, two were eliminated from further analysis because three of four functional scans (namely, V1, V2, V3, or thumb) were not performed because of technical difficulties. These subjects were excluded from further analysis. From the remaining seven subjects, there were 18 usable functional scans after noxious heat applied to the face and four usable functional scans after noxious heat applied to the thumb. Five functional scans were eliminated because of machine [thermal sensory analyzer (TSA)] malfunction $(\phi)$ and one subject (subject 7; Table 3) had significant motion $(\Delta)$ after stimulation to $\mathrm{V} 1$ precluding functional analysis for this stimulus only. Of the 18 facial functional scans, significant activations were observed in the ipsilateral $\mathrm{spV}$ in 15 cases (see Individual analysis below, Table 3).

\section{Activation in spinal nucleus of $\mathbf{V}(\mathbf{s p V})$} Validation of activation in $s p V$

Figure $3 A$ shows an example of signal change in the brainstem of a single subject after a noxious thermal stimulus. Significant activation is present within the brainstem in the region of the $\mathrm{spV}$ ipsilateral to the stimulus. The activation is focal, and there is no activation along the brainstem edge, suggesting the absence of partial volume effects. The volume of activation represents three contiguous pixels. The time course within this region is similar to that in other activated regions of the brain. Activations in nonneuronal tissue outside of the brainstem lack temporal correlation with the thermal stimulus. Evaluation of equivalent volumes of brain tissue (pixels) within the brainstem immediately adjacent to the activation and also on the contralateral side indicates that the signal change drops off significantly in the adjacent pixels and is minimal on the contralateral side (Fig. $3 A$ ).

Further analysis indicated that motion of the brainstem did not contribute significantly to the signal change observed in the spV. Fourier analysis of the temporal variations in the activated area and its surroundings (Fig. $3 B$ ) was performed to evaluate highfrequency residual components that may contribute to the activation. As shown in Figure 3B, the activated area presents a Fourier spectrum with a significant component associated with the delivered stimulus; this component is much larger than the others that are most likely associated with cardiac or respiratory pulsations. 
Table 3. Individual analysis

\begin{tabular}{|c|c|c|c|c|c|c|c|c|c|}
\hline \multirow[b]{2}{*}{ Stimulus site } & \multirow[b]{2}{*}{ Brain region } & \multicolumn{7}{|l|}{ Subject } & \multirow[b]{2}{*}{ Total $^{a}$} \\
\hline & & 1 & 2 & 3 & 4 & 5 & 6 & 7 & \\
\hline \multirow[t]{5}{*}{ Ophthalmic division (V1) } & $\mathrm{spV}$ & $+/(+)$ & $+/(+)$ & $+/(+)$ & $-/(-)$ & $+/(+)$ & $-/(+)$ & $\Delta$ & $(5) / 6$ \\
\hline & Thal & & & & & & & & \\
\hline & VPM/VPL & + & + & + & + & + & - & $\Delta$ & $5 / 6$ \\
\hline & $\mathrm{DM}$ & + & + & + & - & + & - & $\Delta$ & $4 / 6$ \\
\hline & $\mathrm{S} 1$ & + & + & + & + & + & + & $\Delta$ & $6 / 6$ \\
\hline \multirow[t]{5}{*}{ Maxillary division (V2) } & $\mathrm{spV}$ & $+/(+)$ & $+/(+)$ & $+/(+)$ & $+/(+)$ & $+/(-)$ & $-/(+)$ & $+/(+)$ & $(6) / 7$ \\
\hline & Thal & & & & & & & & \\
\hline & VPM/VPL & + & + & + & - & + & + & + & $6 / 7$ \\
\hline & DM & + & + & + & + & + & - & - & $5 / 7$ \\
\hline & $\mathrm{S} 1$ & + & + & + & + & + & + & + & $7 / 7$ \\
\hline \multirow[t]{5}{*}{ Mandibular division (V3) } & $\mathrm{spV}$ & $-/(+)$ & $\phi$ & $\phi$ & $-/(-)$ & $-/(+)$ & $+/(+)$ & $+/(+)$ & $(4) /(5)$ \\
\hline & Thal & & & & & & & & \\
\hline & $\mathrm{VPM} / \mathrm{VPL}$ & + & $\phi$ & $\phi$ & + & + & + & - & $4 / 5$ \\
\hline & $\mathrm{DM}$ & + & $\phi$ & $\phi$ & + & - & + & + & $4 / 5$ \\
\hline & $\mathrm{S} 1$ & + & $\phi$ & $\phi$ & - & - & + & + & $3 / 5$ \\
\hline \multirow[t]{4}{*}{ Thumb } & Thal & & & & & & & & \\
\hline & VPL & + & $\phi$ & $\phi$ & + & $\phi$ & + & + & $4 / 4$ \\
\hline & $\mathrm{DM}$ & + & $\phi$ & $\phi$ & + & $\phi$ & - & + & $3 / 4$ \\
\hline & $\mathrm{S} 1$ & + & $\phi$ & $\phi$ & - & $\phi$ & + & + & $3 / 4$ \\
\hline
\end{tabular}

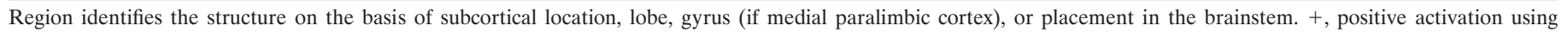
Talairaich; $(+)$, positive activation using the parcellation method; $(-)$, no significant activation; Thal, thalamus.

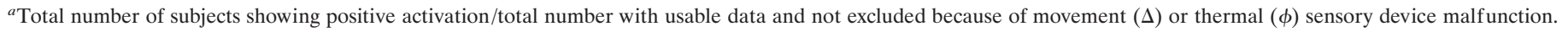

\section{Group analysis ( $s p V)$}

Table 2 shows activation in the $\mathrm{spV}$ after noxious thermal stimulation to V1, V2, and V3 divisions of the face. The data are from six individuals for $\mathrm{V} 1$, seven individuals for $\mathrm{V} 2$, and five individuals for V3. Significant activations were observed within the rostrocaudal extent of the ipsilateral brainstem in the region of the $\mathrm{spV}$. Note that these activations were plotted in the Talairach space, allowing for a consistent approach to averaging (see Materials and Methods). Although the presentations of stimuli to the face were always in the same order for all subjects (V1 followed by V2 followed by V3), no statistical differences in activations within the $\mathrm{spV}$ were observed (Student's $t$ test). Note that there was no activation within the $\mathrm{spV}$ after thermal stimulation of the thumb.

As shown in Figure $4 A$, the activations associated with stimulation of each division could be segregated in a manner that was predicted by the segmental input from each stimulus site. Thus, after V2 stimulation, activation is present at the most rostral extent; after V3 stimulation, activation is in the middle; and the most caudal extent of activation is seen after V1 stimulation (Fig. $4 B$ ). These results are distinct from the classic distribution of V3, $\mathrm{V} 2$, and $\mathrm{V} 1$ in the rostrocaudal extent of the spV. Time courses for activation for each division are also shown in Figure 4.

\section{Individual analysis ( $\mathrm{spV}$ )}

Using the parcellation method described above (see Materials and Methods), activations were evaluated for each individual after stimuli to V1, V2, and V3. Only those activations within the expected area (e.g., B1p in the ipsilateral brainstem) (Fig. 2) were considered positive. Table 3 shows analysis for each individual. Note that individual activations were present in five of six subjects after V1 stimulation (one subject had a movement artifact), six of seven subjects for V2, and four of five subjects for V3 (in two cases there was machine malfunction).
Figure $5 A$ shows activation within the $\mathrm{spV}$ from two individuals after stimulation to the face at V1 and V2 in each case. The location of activation for each individual was mapped onto the native cross section of the brainstem using the parcellation method described above (Fig. $5 B$ ). Details are provided in the figure legend. Note that the distribution of the activation pattern after stimulation at V1, V2, and V3 approximates the relative location of these in the brainstem as shown in Figure $4 B$.

\section{Thalamic activation}

A $46^{\circ} \mathrm{C}$ stimulus applied to the face produced two separate zones of activation, one in the ventrobasal complex and one in the DM nucleus. Thumb stimulation produced activation in a similar region of the DM nucleus and in a region of the ventrobasal complex that was lateral to the activation produced by facial stimulation.

\section{Group analysis (thalamus)}

After stimulation to each division of the trigeminal nerve, significant thalamic activation was present in two regions in each case. The first included an extended activation within the VPM nucleus, and the second was within the DM nucleus (Table 2). Figure $6 A$ shows activation in the thalamus after stimulation to V1, which was similar to that observed in the structure after stimuli to the other two divisions. Figure $6 B$ is an overlap map showing the common areas of activation within the thalamus after stimuli to V1, V2, and V3.

\section{Individual analysis (thalamus)}

After V1 stimulation, five of six and four of six subjects demonstrated activation in VPM and DM nuclei, respectively. V2 stimulation activated the VPM and DM in six of seven and five of seven subjects, respectively, and after V3 stimulation, four of five subjects showed activation in the VPM nucleus, and four of five 

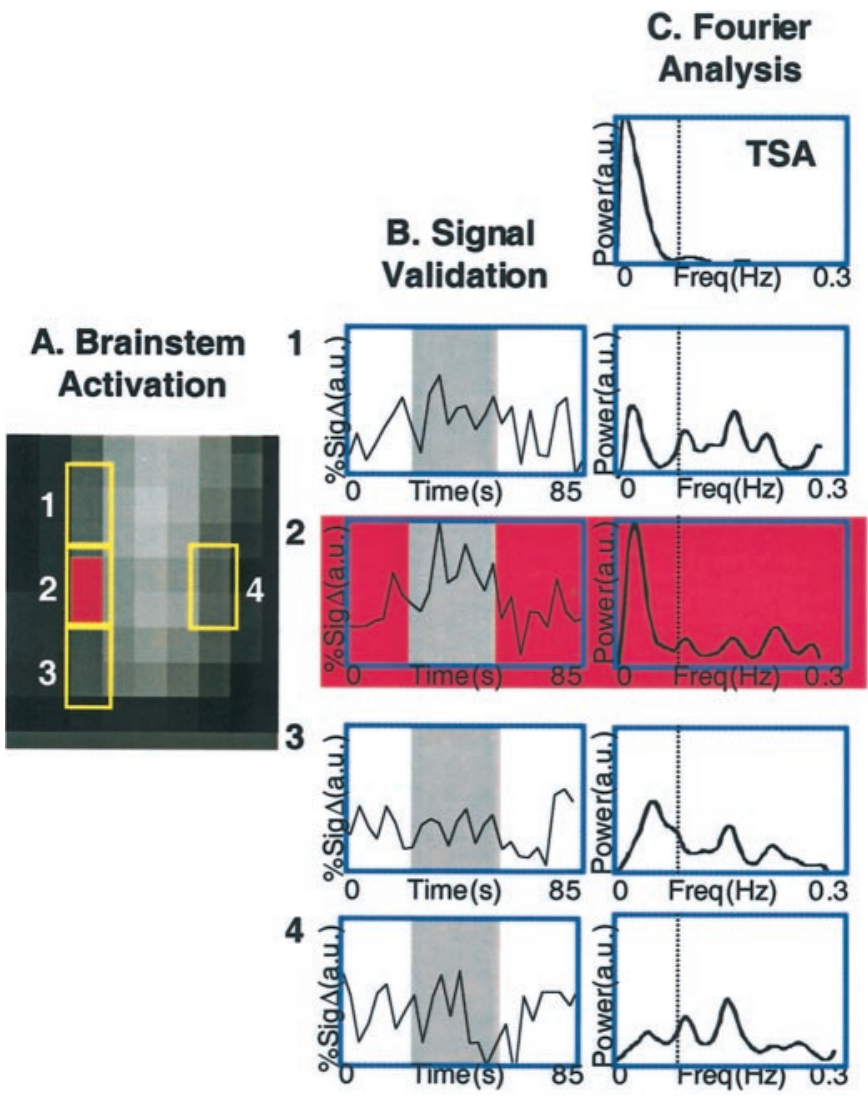

Figure 3. Signal analysis in the brainstem. $A$, Activation map in the brainstem. Shown is the activation map within the brainstem after the $46^{\circ} \mathrm{C}$ stimulus. The area indicated in red (2) is the activated zone, and the panels above (1), below (3), and opposite (4) the area activated (2) are also shown. $B$, Signal validation. The gray region in all the time course graphs indicates the $46^{\circ} \mathrm{C}$ stimulus duration. The panels show time courses of signal change within a three-voxel region, which matches the thermal stimulus temporally. The time courses for activity within brainstem regions of equal volume superior (1), inferior (2), and contralateral (3) to the activated zone (4) are shown. Percent signal change is shown in arbitrary units $($ a.u. $)$. $C$, Fourier analysis. Shown is Fourier analysis for the thermal signal itself (TSA; top panel) and for the adjacent and contralateral signal changes shown in $A$ for brainstem sites $1-4$. The Fourier analyses indicate that other frequency changes are present, which may be respiratory or cardiac. Note, however, that contributions to the signal are present but not significant in the voxels above and below the activation.

showed activation in the DM nucleus (Table 3). As noted in Table 3 , some individuals showed no activation $(-)$ or were excluded because of movement $(\Delta)$ or machine malfunction $(\phi)$.

\section{Thumb (thalamus)}

Application of a painful thermal stimulus to the palmar aspect of the ipsilateral thumb produced thalamic activation in two separate regions that correspond to the VPL and DM nuclei, based on the atlas of Talairach and Tournoux (1988). The activation coordinates (see Table 2) show that this region is lateral to the regions activated by facial stimuli.

\section{S1 activation}

Group analysis (S1)

Significant activation was present in S1 after stimulation to each of the three divisions (Table 2). An overlap map of activation is shown in Figure 7. Note that the distribution of activation follows a pattern that would be predicted by the segmental nature of facial innervation: the rostral components of the face activate S1 regions inferior to those activated by more caudal facial components.

\section{Individual analysis (S1)}

In the S1 cortex, six of sic, seven of seven, and three of five individuals demonstrated activation in the S1 cortex after thermal stimulation to V1, V2, and V3 divisions, respectively.

\section{Thumb (S1)}

Activation in S1 after stimulation to the thumb was present in three of four individuals (in three individuals there was TSA malfunction). The region of activation was adjacent to that observed after stimulation to the most rostral $\mathrm{S} 1$ cortical activation of the face (i.e., V1 and V3) (Fig. 7C).

\section{DISCUSSION}

Noxious stimulation of the face produced somatotopically organized patterns of neural activation detected by fMRI at three levels of the trigeminothalamocortical pathway: the ipsilateral brainstem trigeminal complex, the contralateral somatosensory thalamus, and the contralateral primary somatosensory cortex.

\section{Spinal trigeminal nucleus}

Because electrophysiological mapping of the trigeminal nucleus has not been done in humans, we used anatomical landmarks easily observed in standard MRI images to parcellate the brainstem in a manner that defines the region containing the $\mathrm{spV}$ (see Materials and Methods). The location of activations can be defined within these prescribed anatomical segments (see Materials and Methods). The activated regions in the spV extended along a column ipsilateral to the thermal stimulation in all seven subjects.

After noxious thermal stimuli applied separately to the skin of the face at sites within the distribution of each of the three divisions of the trigeminal nerve, we observed significant activation in the $\mathrm{spV}$ for at least two of the three divisions in all individuals tested. The somatotopy of $\mathrm{spV}$ activation after painful stimuli to the face is in agreement with data from previous electrophysiological and surgical studies. In detailed mapping studies in animals, the $\mathrm{spV}$ exhibits a precise somatotopy that has two components: (1) the dorsoventral axis of the face (V1-V2$\mathrm{V} 3$ ) is represented in a ventral-to-dorsal sequence; and (2) the rostrocaudal axis of the face is represented in a rostral-to-caudal sequence in spV (Price et al., 1976; Sessle et al., 1986; Strassman and Vos, 1993; Strassman et al., 1994). Surgical studies indicate that a similar somatotopy may be present in humans. After trigeminal tractotomy (caudotomy) at different levels, patients develop sensory deficits in the face that vary according to an onion skin or segmental distribution: the perioral areas are represented rostrally in the nucleus, and more posterior (caudal) areas of the face are represented more caudally within the spV (Kunc, 1970). The three facial stimulation sites we used were all located in different zones of the onion skin distribution, and the resulting regions of activation in the brainstem followed a rostrocaudal sequence that could be predicted from their relative positions on the onion skin map. Differences were not observed in the dorsoventral position of the regions of activation. This may be the result of differences between human and animal trigeminal anatomy or most likely a result of technical limitations of spatial resolution of fMRI.

The extent of activation observed in the medullary dorsal horn and caudal pons corresponds with the known distribution of 


\section{A. Predicted Activation in spV}
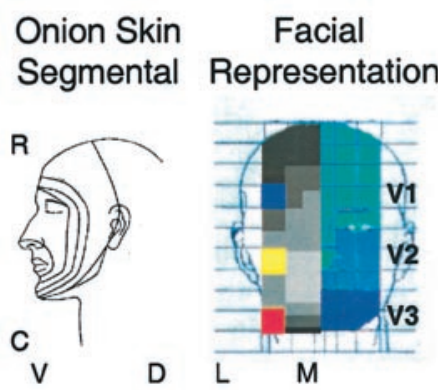

Face Segmental

$\mathrm{spV}$
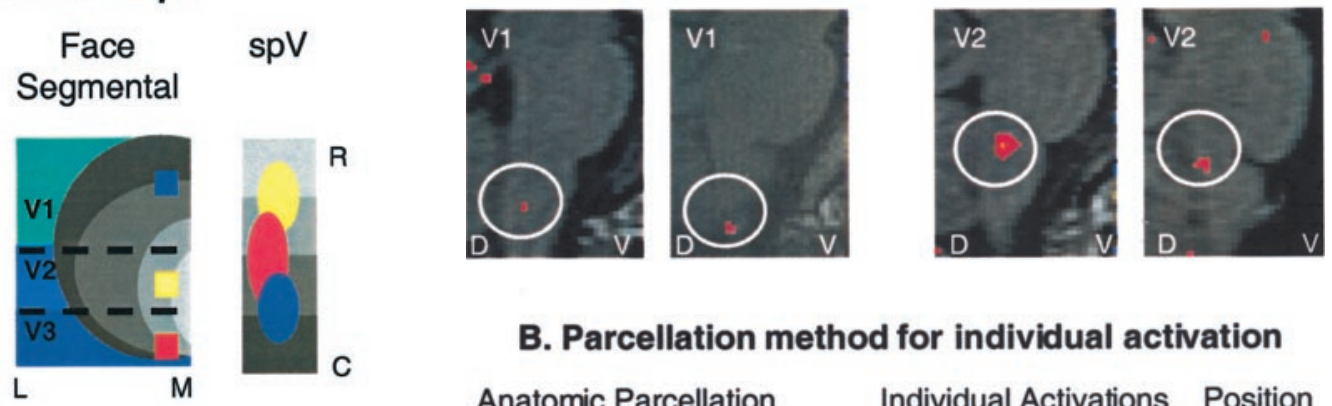

\section{B. Parcellation method for individual activation}

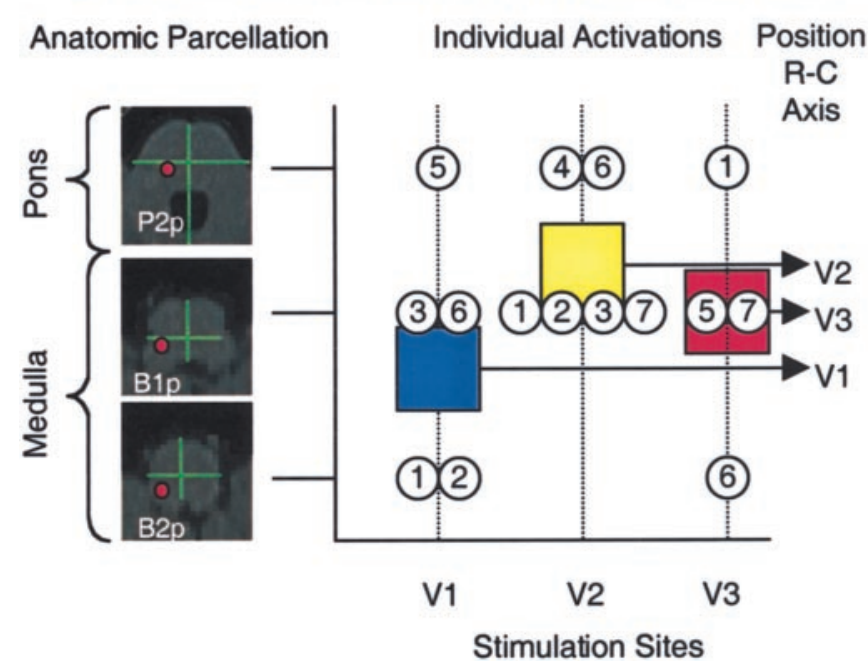

Figure 5. Individual analysis in the spV. A, Activation maps from single subjects. Shown are activation foci from two individuals mapped onto anatomical sagittal sections through the brainstem after a thermal stimulus to V1 or V2. $D$, Dorsal; $V$, ventral. $B$, Individual activations based on the parcellation method of the brainstem. Left panel, The parcellation for the pons and medulla is shown on the left. The red circles represent the expected areas containing the $\mathrm{spV}$ in $P 2 p$ (caudal pons), B1p (rostral medulla), and $B 2 p$ (caudal medulla). Right panel, Individual activations after stimulation to facial sites V1, V2, and V3 were localized according to the brainstem level using the parcellation method shown in the left panel. The mean frequency of activations, plotted as squares, shows the same rostrocaudal $(R-C)$ somatotopy as for group average activations using a different approach as shown in Figure 4B. Blue square, V1; yellow square, $\mathrm{V} 2$; red square, $\mathrm{V} 3$.

nociceptive neurons in all three divisions of $\mathrm{spV}$ in animals: the caudalis, interpolaris, and oralis (Hu et al., 1981; Young and Perryman, 1986; Jacquin and Rhoades, 1990; Raboisson et al., 1995; Dallel et al., 1998; Ro and Capra, 1999). Recordings of

$\leftarrow$

segments in the rostrocaudal extent as well as three different divisions of the trigeminal nerve. $R$, Rostral; $C$, caudal; $L$, lateral; $M$, medial; $V$, ventral; $D$, dorsal. $B$, Actual activations in the $\mathrm{spV}$. Shown is group average activation in $\mathrm{spV}$ after thermal stimulation to V1, V2, and V3 divisions of the trigeminal nerve. The leftmost panel is an overlay of statistical maps of averaged data from seven healthy subjects after $46^{\circ} \mathrm{C}$ stimulation with a $1.6 \times 1.6 \mathrm{~cm}$ Peltier thermode to V1, V2, and V3 of the face. Note that these are actual data, but that the regions of activation are color-coded for each area stimulated $(p<0.005)$. The center panels show statistical maps of activation in the sagittal and horizontal planes in the Talairach domain. The use of parcellation methods allows for definition of activation within a region of the brainstem correlating with the location of the trigeminal nucleus. Time courses for each group are also shown (right panels). The color code for each corresponds with the overlap map of activations within the nucleus. $V$, Ventral; $D$, dorsal; numbers in the top right corners indicate coordinates in the mediolateral axis (left panel) or in the rostrocaudal $(z)$ axis. 


\section{A. Group Activation}

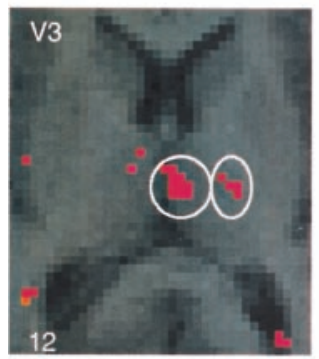

$3.2 \times 10^{-4} 1 \times 10^{-6}$

\section{B. Face and Thumb Activation}

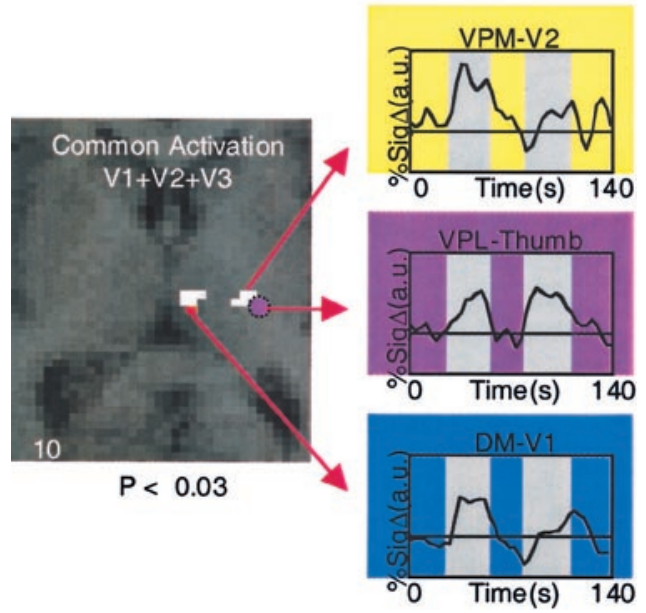

Figure 6. Activation in the thalamus. A, Group activation. Activation in the contralateral thalamus is shown after a noxious thermal stimulus to the V3 region of the face. $B$, Face and thumb activation. Activation is shown in the contralateral thalamus after stimulation to the face and hand. The white areas show regions of common activation after thermal stimulation to the V1, V2, and V3 distributions of the face in regions defined as the DM and VPM nuclei. Activation of the thumb is mapped onto the same anatomical section (purple circle) and corresponds to the VPL nucleus. The regions are defined anatomically using the atlas of Talairach and Tournoux (1988). Time courses of activation for each area are shown in the insets. Percent signal change is shown in arbitrary units (a.u.); numbers in the bottom corners indicate the Talairach coordinates in the rostrocaudal $(z)$ axis. nociceptive neurons in human $\mathrm{spV}$ have not been made; however, indirect evidence suggests a similar distribution (Ellrich et al., 1999).

\section{Thalamus}

A number of fMRI studies have demonstrated activation in the human thalamus (Davis et al., 1998; Becerra et al., 1999; 2001; Peyron et al., 2000). The spinothalamic tract conveys nociceptive information from the dorsal horn and trigeminal nucleus to nuclei within the thalamus, including the ventrobasal complex (Dostrovsky, 2000). Classic descriptions of the somatotopy show that the face is represented in the VPM nucleus and the body is represented in the VPL nucleus (Casey and Morrow, 1983; Chung et al., 1986; Apkarian et al., 2000b; Craig and Dostrovsky, 2001). However, human imaging studies have not differentiated between activation in the VPM and VPL nuclei after noxious stimuli to the face and body. Our data show that all facial stimuli produce peak activation in a region corresponding to the VPM nucleus, whereas thumb stimulation activates a region corresponding to the VPL nucleus. After both facial and thumb stimuli, we observe activation in the DM nucleus as shown previously after a noxious thermal stimulus to the dorsum of the hand (Becerra et al., 1999). This area may correspond to the region of the thalamus that has specific thermoreceptive neurons (Craig et al., 1999; Blomqvist et al., 2000).

Data from the animal (Yokota et al., 1985; Rausell and Jones, 1991; Koyama et al., 1998) and human (Lenz et al., 1988) literature have defined somatotopic activation in the thalamus with different facial sites. The maps obtained in these electrophysiological studies show that all three of the stimulation sites used in the present study would produce activation primarily within the dorsal part of the VPM nucleus, whereas the ventral part of the nucleus contains the intraoral representation. In this study, there was no obvious somatotopy in the thalamic activation produced by V1, V2, or V3 stimulation. This could be attributable to the proximate input from the three stimulation sites within VPM nucleus and the limitations of resolution using a $1.5 \mathrm{~T}$ magnet.

\section{Primary somatosensory cortex}

Physiological and anatomical data have demonstrated somatotopic representation in S1 (Merzenich et al., 1978; Kaas, 1983; Garraghty et al., 1990). Recent neuroimaging studies have demonstrated varying degrees of somatotopic activation in S1 after

\section{A. Facial B. Face Representation Segmental}

C. S1 Cortex

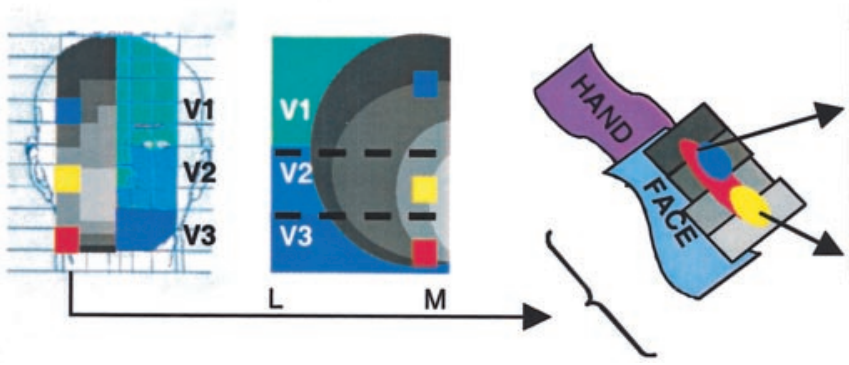

D. Cortical Activation

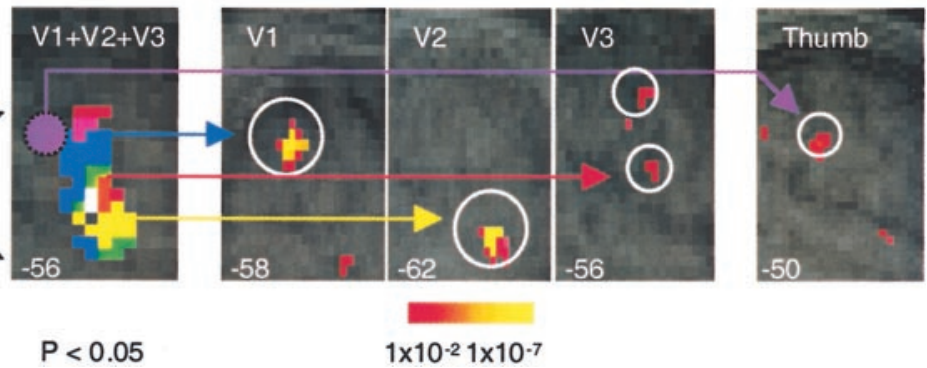

Figure 7. Activation in S1. A, Facial representation (see legend to Fig. 4A, second panel). B, Face, segmental (see legend to Fig. 4A, third panel). $C$, $\mathrm{S} 1$ cortex. Shown is the expected activation patterns in a schematic of the S1 cortex based on the segmental nature of the face and the specific sites stimulated (shown in $B$ ). $D$, Cortical activation maps. Shown is activation in the contralateral somatosensory cortex after $46^{\circ} \mathrm{C}$ stimulus to the right V1, V2, and V3 divisions of the trigeminal nerve $(A)$ and right thumb in seven subjects. Activations are shown as statistical maps of the contralateral cortex, color-coded to match the stimulation sites for each region in $C$. C, Composite of activations. $P$, Posterior; A, anterior; numbers in the bottom corners indicate the Talairach coordinate in the mediolateral $(x)$ axis. 
sensory stimuli principally to the hand and fingers (Lin et al., 1996; Gelnar et al., 1998; Kurth et al., 1998; Hlustik et al., 2001). In addition, using optical imaging of the human cortex during surgery, one study has reported segregation of cortical activation after electrical stimulation of the first and third divisions of the trigeminal nerve (Sato et al., 2002). In the current study, the pattern of activation produced in S1 by stimulation of the three facial sites showed greater overlap than in the brainstem but still demonstrated clear somatotypy with an inverted representation of the face: the most caudal facial site (V1) was closer to the hand area than the most rostral facial site (V2). The two components that are farthest apart in the brainstem are also farthest apart in the cortex, with the caudal part of the face being closest to the thumb. Some potential confusion is caused by the fact that activation after V3 stimulation occurs in two areas in S1: one in the middle portion of the column and one in the rostral extent. A similar pattern of isolated "islands" of activation within the cortex after facial stimulation in monkeys has been shown using electrophysiology (Jain et al., 2001). The presence of V3 activation both above and below V1 activation may explain the controversy that exists over whether the representation of the face in S1 is right side-up (Penfield and Rasmussen, 1950) or inverted (Servos et al., 1999). The pattern of V3 activation may depend on the site of facial stimulation relative to the onion skin segmental arrangement.

S1 activation after noxious stimulation of the thumb was located adjacent and dorsorostral to the area activated by V1 and V3, in agreement with the classical Penfield description of S1 organization and more recent fMRI studies of S1 activation in humans (Moore et al., 2000) and monkeys (Hayashi et al., 1999). The use of cortical flattening techniques (Dale et al., 1999; Fischl et al., 1999) will allow for further definition of these sensory maps.

\section{Caveats}

A number of caveats should be raised in connection with this study. Although the anatomy of the human trigeminal system has been defined, its functional organization has not. Data from nonhuman primates and from surgical interventions in patients have shown that the basic organization is similar to that of the rodent (Kunc 1970; Afshar and Dykes, 1984; Brown, 1997; Usunoff et al., 1997; Jawahar et al., 2001). The data presented here suggest that functional changes occur within the $\mathrm{spV}$ based on where the face is stimulated relative to the dermatomal pattern.

A second issue relates to ordering effects. The aim of these experiments was to localize $\mathrm{spV}$ activation after stimulation to each of the three divisions of the trigeminal nerve. We believe that the order of stimulation in our approach is unlikely to affect localization of activation; it may effect the intensity of activation. The VAS data show that there is a small change in VAS across the three sites with V2 $>$ V1 $>$ V3 for the same thermal stimulus. In previous studies using thermal stimuli to the hand, we found that four sequential stimuli in relatively rapid succession produced adaptation (Becerra et al., 1999, 2001; Zubieta et al., 2001). The current paradigm differs in two important respects: only two stimuli were administered to each site, and there was a separation of $\sim 3$ min between stimulation at different sites. There was no significant difference in percent signal change for the three sites (see Results), suggesting that the paradigm did not produce central sensitization at one site, affecting another.

\section{Conclusions}

This study demonstrates the ability to simultaneously measure changes at three levels of the trigeminal sensory pathway (spV-
thalamus-S1 cortex) in individual subjects after a noxious stimulus to the skin of the face. The ability to measure functional changes within spV using fMRI will allow a number of important developments, including mapping of the nucleus in more detail using higher-field magnets and the ability to evaluate altered activity at the level of the first nociceptive synapse in surrogate models of pain and in human subjects with clinical pain. Furthermore, these data suggest that an objective fMRI-based method can be developed for testing the efficacy of analgesics in clinical pain states.

\section{REFERENCES}

Afshar F, Dykes E (1984) Computer-generated three-dimensional visualization of the trigeminal nuclear complex. Surg Neurol 22:189-196.

Apkarian AV, Gelnar PA, Krauss BR, Szeverenyi NM (2000a) Cortical responses to thermal pain depend on stimulus size: a functional MRI study. J Neurophysiol 83:3113-3122.

Apkarian AV, Shi T, Bruggemann J, Airapetian LR (2000b) Segregation of nociceptive and non-nociceptive networks in the squirrel monkey somatosensory thalamus. J Neurophysiol 84:484-494.

Becerra LR, Breiter HC, Stojanovic M, Fishman S, Edwards A, Comite AR, Gonzalez RG, Borsook D (1999) Human brain activation under controlled thermal stimulation and habituation to noxious heat: an fMRI study. Magn Reson Med 41:1044-1057.

Becerra L, Breiter HC, Wise R, Gonzalez RG, Borsook D (2001) Reward circuitry activation by noxious thermal stimuli. Neuron 32:927-946.

Blomqvist A, Zhang ET, Craig AD (2000) Cytoarchitectonic and immunohistochemical characterization of a specific pain and temperature relay, the posterior portion of the ventral medial nucleus, in the human thalamus. Brain 123:601-619.

Brown JA (1997) The trigeminal complex: anatomy and physiology. Neurosurg Clin North Am 8:1-10.

Bushnell MC, Duncan GH, Dubner R, He LF (1984) Activity of trigeminothalamic neurons in medullary dorsal horn of awake monkeys trained in a thermal discrimination task. J Neurophysiol 52:170-187.

Bushnell MC, Duncan GH, Tremblay N (1993) Thalamic VPM nucleus in the behaving monkey. I. Multimodal and discriminative properties of thermosensitive neurons. J Neurophysiol 69:739-752.

Bushnell MC, Duncan GH, Hofbauer RK, Ha B, Chen JI, Carrier B (1999) Pain perception: is there a role for primary somatosensory cortex? Proc Natl Acad Sci USA 96:7705-7709.

Carpenter MB, Sutin J (1983) Human neuroanatomy. Baltimore: Williams \& Wilkins.

Casey KL, Morrow TJ (1983) Ventral posterior thalamic neurons differentially responsive to noxious stimulation of the awake monkey. Science 221:675-677.

Casey KL, Minoshima S, Morrow TJ, Koeppe RA (1996) Comparison of human cerebral activation pattern during cutaneous warmth, heat pain, and deep cold pain. J Neurophysiol 76:571-581.

Chudler EH, Anton F, Dubner R, Kenshalo Jr DR (1990) Responses of nociceptive S1 neurons in monkeys and pain sensation in humans elicited by noxious thermal stimulation: effect of interstimulus interval. J Neurophysiol 63:559-569.

Chung JM, Lee KH, Surmeier DJ, Sorkin LS, Kim J, Willis WD (1986) Response characteristics of neurons in the ventral posterior lateral nucleus of the monkey thalamus. J Neurophysiol 56:370-390.

Coghill RC, Talbot JD, Evans AC, Meyer E, Gjedde A, Bushnell MC, Duncan GH (1994) Distributed processing of pain and vibration by the human brain. J Neurosci 14:4095-4108.

Coghill RC, Sang CN, Maisog JM, Iadarola MJ (1999) Pain intensity processing within the human brain: a bilateral, distributed mechanism. J Neurophysiol 82:1934-1943.

Coghill RC, Gilron I, Iadarola MJ (2001) Hemispheric lateralization of somatosensory processing. J Neurophysiol 85:2602-2612.

Craig AD, Dostrovsky JO (2001) Differential projections of thermoreceptive and nociceptive lamina I trigeminothalamic and spinothalamic neurons in the cat. J Neurophysiol 86:856-870.

Craig AD, Zhang ET, Blomqvist A (1999) A distinct thermoreceptive subregion of lamina I in nucleus caudalis of the owl monkey. J Comp Neurol 404:221-234.

Dallel R, Duale C, Molat JL (1998) Morphine administered in the substantia gelatinosa of the spinal trigeminal nucleus caudalis inhibits nociceptive activities in the spinal trigeminal nucleus oralis. J Neurosci 18:3529-3536.

DaSilva AFM, Becerra L, Geatrakas C, Strassman A, Makris N, Keith D, Gonzalez G, Breiter H, Borsook D (2001) fMRI activation in the trigeminal pain pathways (spV, thalamus, SI) following noxious heat. Soc Neurosci Abstr 27:1328.

Dale AM, Fischl B, Sereno MI (1999) Cortical surface-based analysis. I. Segmentation and surface reconstruction. NeuroImage 9:179-194. 
Davis KD, Kwan CL, Crawley AP, Mikulis DJ (1998) Functional MRI study of thalamic and cortical activations evoked by cutaneous heat, cold, and tactile stimuli. J Neurophysiol 80:1533-1546.

Dostrovsky JO (2000) Role of thalamus in pain. Prog Brain Res 129:245-257.

Dostrovsky JO, Guilbaud G (1990) Nociceptive responses in medial thalamus of the normal and arthritic rat. Pain 40:93-104.

Dubner R, Kenshalo Jr DR, Maixner W, Bushnell MC, Oliveras JL (1989) The correlation of monkey medullary dorsal horn neuronal activity and the perceived intensity of noxious heat stimuli. J Neurophysiol 62:450-457.

Ellrich J, Andersen OK, Messlinger K, Arendt-Nielsen L (1999) Convergence of meningeal and facial afferents onto trigeminal brainstem neurons: an electrophysiological study in rat and man. Pain 82:229-237.

Fischl B, Sereno MI, Dale AM (1999) Cortical surface-based analysis II: inflation, flattening, and a surface-based coordinate system. NeuroImage 9:195-207.

Forman SD, Cohen JD, Fitzgerald M, Eddy WF, Mintun MA, Noll DC (1995) Improved assessment of significant activation in functional magnetic resonance imaging (fMRI): use of a cluster-size threshold. Magn Reson Med 33:636-647.

Garraghty PE, Florence SL, Kaas JH (1990) Ablations of areas 3a and $3 \mathrm{~b}$ of monkey somatosensory cortex abolish cutaneous responsivity in area 1. Brain Res 528:165-169.

Gelnar PA, Krauss BR, Szeverenyi NM, Apkarian AV (1998) Fingertip representation in the human somatosensory cortex: an fMRI study. NeuroImage 7:261-283.

Gybels JM, Sweet WH (1989) In: Neurosurgical treatment of persistent pain. Basel: Karger.

Haines D (1994) Neuroanatomy: an atlas of structures, sections, and systems. Baltimore: Williams \& Wilkins.

Hayashi T, Konishi S, Hasegawa I, Miyashita Y (1999) Short communication: mapping of somatosensory cortices with functional magnetic resonance imaging in anaesthetized macaque monkeys. Eur J Neurosci 11:4451-4456.

Hlustik P, Solodkin A, Gullapalli RP, Noll DC, Small SL (2001) Somatotopy in human primary motor and somatosensory hand representations revisited. Cereb Cortex 11:312-321.

Hofbauer RK, Rainville P, Duncan GH, Bushnell MC (2001) Cortical representation of the sensory dimension of pain. J Neurophysiol 86:402-411.

Hsieh JC, Hannerz J, Ingvar M (1996) Right-lateralised central processing for pain of nitroglycerin-induced cluster headache. Pain 67:59-68.

Hu JW, Dostrovsky JO, Sessle BJ (1981) Functional properties of neurons in cat trigeminal subnucleus caudalis (medullary dorsal horn). I. Responses to oral-facial noxious and nonnoxious stimuli and projections to thalamus and subnucleus oralis. J Neurophysiol 45:173-192.

Jacquin MF, Rhoades RW (1990) Cell structure and response properties in the trigeminal subnucleus oralis. Somatosens Mot Res 7:265-288.

Jain N, Qi HX, Catania KC, Kaas JH (2001) Anatomic correlates of the face and oral cavity representations in the somatosensory cortical area 3b of monkeys. J Comp Neurol 429:455-468.

Jawahar A, Kondziolka D, Kanal E, Bissonette DJ, Lunsford LD (2001) Imaging the trigeminal nerve and pons before and after surgical intervention for trigeminal neuralgia. Neurosurgery 48:101-106.

Kaas JH (1983) What, if anything, is SI? Organization of first somatosensory area of cortex. Physiol Rev 63:206-231.

Kennedy DN, Makris N, Bates JF, Caviness Jr VS (1997) Structural morphometry of the developing brain. In: Developmental neuroimaging: mapping the development of brain and behavior (Thatcher RW, Lyon GR, Rumsey J, Krasnegor N, eds), pp 29-41. San Diego: Academic.

Kim JS, Lee JH, Lee MC (1997) Patterns of sensory dysfunction in lateral medullary infarction. Clinical-MRI correlation. Neurology 49:1557-1563.

Koyama N, Nishikawa Y, Yokota T (1998) Distribution of nociceptive neurons in the ventrobasal complex of macaque thalamus. Neurosci Res 31:39-51.

Kunc Z (1970) Significant factors pertaining to the results of trigeminal tractotomy. In: Trigeminal neuralgia, pathogenesis and pathophysiology (Hassler R, Walker AE, eds), pp 90-100. Stuttgart: Thieme.

Kurth R, Villringer K, Mackert BM, Schwiemann J, Braun J, Curio G, Villringer A, Wolf KJ (1998) fMRI assessment of somatotopy in human Brodmann area $3 \mathrm{~b}$ by electrical finger stimulation. NeuroReport 9:207-212.

Lenz FA, Dostrovsky JO, Tasker RR, Yamashiro K, Kwan HC, Murphy JT (1988) Single-unit analysis of the human ventral thalamic nuclear group: somatosensory responses. J Neurophysiol 59:299-316.

Lin W, Kuppusamy K, Haacke EM, Burton H (1996) Functional MRI in human somatosensory cortex activated by touching textured surfaces. J Magn Reson Imaging 6:565-572.

Maixner W, Dubner R, Kenshalo Jr DR, Bushnell MC, Oliveras JL
(1989) Responses of monkey medullary dorsal horn neurons during the detection of noxious heat stimuli. J Neurophysiol 62:437-449.

Manger PR, Woods TM, Jones EG (1996) Representation of face and intra-oral structures in area $3 \mathrm{~b}$ of macaque monkey somatosensory cortex. J Comp Neurol 371:513-521.

May A, Kaube H, Buchel C, Eichten C, Rijntjes M, Juptner M, Weiller C, Diener HC (1998) Experimental cranial pain elicited by capsaicin: a PET study. Pain 74:61-66.

Merzenich MM, Kaas JH, Sur M, Lin CS (1978) Double representation of the body surface within cytoarchitectonic areas $3 b$ and 1 in "SI" in the owl monkey (Aotus trivirgatus). J Comp Neurol 181:41-73.

Moore CI, Stern CE, Corkin S, Fischl B, Gray AC, Rosen BR, Dale AM (2000) Segregation of somatosensory activation in the human rolandic cortex using fMRI. J Neurophysiol 84:558-569.

Penfield W, Rasmussen T (1950) Secondary sensory and motor representation. In: The cerebral cortex of man, pp 109-134 New York: Macmillan.

Peyron R, Laurent B, Garcia-Larrea L (2000) Functional imaging of brain responses to pain. A review and meta-analysis. Neurophysiol Clin 30:263-288

Price DD, Dubner R, Hu JW (1976) Trigeminothalamic neurons in nucleus caudalis responsive to tactile, thermal, and nociceptive stimulation of monkey's face. J Neurophysiol 39:936-953.

Raboisson P, Dallel R, Clavelou P, Sessle BJ, Woda A (1995) Effects of subcutaneous formalin on the activity of trigeminal brain stem nociceptive neurones in the rat. J Neurophysiol 73:496-505.

Rausell E, Jones EG (1991) Chemically distinct compartments of the thalamic VPM nucleus in monkeys relay principal and spinal trigeminal pathways to different layers of the somatosensory cortex. J Neurosci $11: 226-237$

Ro JY, Capra NF (1999) Evidence for subnucleus interpolaris in craniofacial muscle pain mechanisms demonstrated by intramuscular injections with hypertonic saline. Brain 842:166-183.

Sato K, Nariai T, Sasaki S, Yazawa I, Mochida H, Miyakawa N, MomoseSato Y, Kamino K, Ohta Y, Hirakawa K, Ohno K (2002) Intraoperative intrinsic optical imaging of neuronal activity from subdivisions of the human primary somatosensory cortex. Cereb Cortex 12:269-280.

Servos P, Engel SA, Gati J, Menon R (1999) fMRI evidence for an inverted face representation in human somatosensory cortex. NeuroReport 10:1393-1395.

Sessle BJ, Hu JW, Amano N, Zhong G (1986) Convergence of cutaneous, tooth pulp, visceral, neck and muscle afferents onto nociceptive and non-nociceptive neurones in trigeminal subnucleus caudalis (medullary dorsal horn) and its implications for referred pain. Pain 27:219-235.

Sherman SE, Luo L, Dostrovsky JO (1997) Spinal strychnine alters response properties of nociceptive-specific neurons in rat medial thalamus. J Neurophysiol 78:628-637.

Strassman AM, Vos BP (1993) Somatotopic and laminar organization of fos-like immunoreactivity in the medullary and upper cervical dorsal horn induced by noxious facial stimulation in the rat. J Comp Neurol $331: 495-516$

Strassman AM, Mineta Y, Vos BP (1994) Distribution of fos-like immunoreactivity in the medullary and upper cervical dorsal horn produced by stimulation of dural blood vessels in the rat. J Neurosci 14:3725-3735.

Svensson P, Minoshima S, Beydoun A, Morrow TJ, Casey KL (1997) Cerebral processing of acute skin and muscle pain in humans. J Neurophysiol 78:450-460.

Talairach J, Tournoux P (1988) Co-planar stereotaxic atlas of the human brain. Stuttgart: Thieme.

Talbot JD, Marrett S, Evans AC, Meyer E, Bushnell MC, Duncan GH (1991) Multiple representations of pain in human cerebral cortex. Science 251:1355-1358.

Usunoff KG, Marani E, Schoen JH (1997) The trigeminal system in man. Adv Anat Embryol Cell Biol 136:1-126.

Weiller C, May A, Limmroth V, Juptner M, Kaube H, Schayck RV, Coenen HH, Diener HC (1995) Brain stem activation in spontaneous human migraine attacks. Nat Med 1:658-660.

Williams MN, Zahm DS, Jacquin MF (1994) Differential foci and synaptic organization of the principal and spinal trigeminal projections to the thalamus in the rat. Eur J Neurosci 6:429-453.

Yokota T, Koyama N, Matsumoto N (1985) Somatotopic distribution of trigeminal nociceptive neurons in ventrobasal complex of cat thalamus. J Neurophysiol 53:1387-1400.

Young RF, Perryman KM (1986) Neuronal responses in rostral trigeminal brain-stem nuclei of macaque monkeys after chronic trigeminal tractotomy. J Neurosurg 65:508-516.

Zubieta JK, Smith YR, Bueller JA, Xu Y, Kilbourn MR, Jewett DM, Meyer CR, Koeppe RA, Stohler CS (2001) Regional $\mu$ opioid receptor regulation of sensory and affective dimensions of pain. Science 293:311-315. 